\title{
Topologically Convergent and Divergent Structural Connectivity Patterns between Patients with Remitted Geriatric Depression and Amnestic Mild Cognitive Impairment
}

\author{
Feng Bai, ${ }^{1,2 *} \mathrm{Ni}$ Shu, ${ }^{3 *}$ Yonggui Yuan, ${ }^{1}$ Yongmei Shi, ${ }^{1}$ Hui Yu, ${ }^{2}$ Di Wu, ${ }^{2}$ Jinhui Wang, ${ }^{3}$ Mingrui Xia, ${ }^{3}$ Yong He, ${ }^{3}$ \\ and Zhijun Zhang ${ }^{1}$ \\ ${ }^{1}$ Department of Neurology, Affiliated ZhongDa Hospital of Southeast University, and Institute of Neuropsychiatry of Southeast University, and ${ }^{2}$ Medical \\ School of Southeast University, Nanjing 210009, China, and ${ }^{3}$ State Key Laboratory of Cognitive Neuroscience and Learning, Beijing Normal University, \\ Beijing 100875, China
}

\begin{abstract}
Alzheimer's disease (AD) can be conceptualized as a disconnection syndrome. Both remitted geriatric depression (RGD) and amnestic mild cognitive impairment (aMCI) are associated with a high risk for developing AD. However, little is known about the similarities and differences in the topological patterns of white matter (WM) structural networks between RGD and aMCI. In this study, diffusion tensor imaging and deterministic tractography were used to map the human WM networks of 35 RGD patients, 38 aMCI patients, and 30 healthy subjects. Furthermore, graph theoretical methods were applied to investigate the alterations in the global and regional properties of the WM network in these patients. First, both the RGD and aMCI patients showed abnormal global topology in their WM networks (i.e., reduced network strength, reduced global efficiency, and increased absolute path length) compared with the controls, and there were no significant differences in these global network properties between the patient groups. Second, similar deficits of the regional and connectivity characteristics in the WM networks were primarily found in the frontal brain regions of RGD and aMCI patients compared with the controls, while a different nodal efficiency of the posterior cingulate cortex and several prefrontal brain regions were also observed between the patient groups. Together, our study provides direct evidence for the association of a great majority of convergent and a minority of divergent connectivity of WM structural networks between RGD and aMCI patients, which may lead to increasing attention in defining a population at risk of AD.
\end{abstract}

\section{Introduction}

Late-onset depression, a clinical syndrome associated with an increased risk for developing Alzheimer's disease (AD) (Blasko et al., 2010), is complicated by cognitive impairments, including marked or mild impairment in cognitive function (Butters et al., 2004). Moreover, depressive symptoms and cognitive impairments are thought to have interactive effects (Goveas et al., 2011; Xie et al., 2011). However, whether these depressive symptoms constitute a true risk factor or are a consequence of the cognitive decline that leads to $\mathrm{AD}$ remains largely unclear.

\footnotetext{
Received 0ct. 6, 2011; revised Jan. 11, 2012; accepted Jan. 25, 2012.

Author contributions: F.B., Y.Y., Y.S., H.Y., and Z.Z. designed research; F.B., Y.Y., Y.S., H.Y., and D.W. performed research; M.X. contributed unpublished reagents/analytic tools; F.B., N.S., J.W., and Y.H. analyzed data; F.B., N.S., Y.H., and Z.Z. wrote the paper.

This work was partly supported by National Natural Science Foundation of China Grants 30825014, 30971016 , $30970814,91132727,81030028,30870667$, and 81000633, and Beijing Natural Science Foundation Grant 7102090. The authors declare no competing financial interests.

*F.B. and N.S. contributed equally to this work.

Correspondence should be addressed to either of the following: Dr. Zhijun Zhang, Department of Neurology, Affiliated ZhongDa Hospital of Southeast University, Nanjing 210009, China, E-mail:zhijunzhang838@yahoo.com.cn; or Dr. Yong He, State Key Laboratory of Cognitive Neuroscience and Learning, Beijing Normal University, Beijing 100875, China, E-mail: yong.he@bnu.edu.cn.

DOI:10.1523/JNEUROSC1.5061-11.2012

Copyright $\odot 2012$ the authors $\quad 0270-6474 / 12 / 324307-12 \$ 15.00 / 0$
}

In first-episode remitted geriatric depression (RGD) subjects, alterations of structure and function have been shown in the frontal-temporal-parietal regions, particularly in the frontal areas (Alexopoulos et al., 2008; Yuan et al., 2008a,b). Specifically, approximately one-half of demonstrated brain $\beta$-amyloid accumulation in the aforementioned cortical areas among RGD is in a pattern characteristic of early AD (Butters et al., 2008). Such a changing pattern was also observed in amnestic mild cognitive impairment (aMCI) pathologic (Jagust et al., 2010) and neuroimaging (Pihlajamäki et al., 2009) studies. aMCI has a probability of developing into $\mathrm{AD}$ with a prevalence of $10-15 \%$ per year (Petersen et al., 1999), and a combination of aMCI and depression has been thought to represent the superimposed AD neuropathology (Butters et al., 2008). In particular, the predominance of cognitive impairments in aMCI involves memory dysfunction (Petersen et al., 1999), while RGD is particularly associated with dysfunction in executive control (Morimoto et al., 2011). Thus, we assume that there are commonalities and distinctions in the neurophysiologic mechanisms in RGD and aMCI.

The biological hypothesis of $\mathrm{AD}$ as a disconnection syndrome have been proposed, involving progressive biochemical and structural changes, which begin at the cellular and synaptic level and ultimately culminate in neuronal death and white matter 
Table 1. Cortical and subcortical regions of interest defined in the study

\begin{tabular}{lllll}
\hline Index & Regions & Abbreviation & Index & Regions \\
\hline$(1,2)$ & Precental gyrus & PreCG & $(47,48)$ & Lingual gyrus \\
$(3,4)$ & Superior frontal gyrus, dorsolateral & SFGdor & $(49,50)$ & Superior occipital gyrus \\
$(5,6)$ & Superior frontal gyrus, orbital part & ORBsup & $(51,52)$ & Middle occipital gyrus \\
$(7,8)$ & Middle frontal gyrus & MFG & $(53,54)$ & Inferior occipital gyrus \\
$(9,10)$ & Middle frontal gyrus, orbital part & ORBmid & $(55,56)$ & Fusiform gyrus \\
$(11,12)$ & Inferior frontal gyrus, opercular part & IFGoperc & $(57,58)$ & Postcentral gyrus \\
$(13,14)$ & Inferior frontal gyrus, triangular part & IFGtriang & $(59,60)$ & Superior parietal gyrus \\
$(15,16)$ & Inferior frontal gyrus, orbital part & ORBinf & $(61,62)$ & Inferior parietal, but supramarginal and angular gyri \\
$(17,18)$ & Rolandic operculum & ROL & $(63,64)$ & Supramarginal gyrus \\
$(19,20)$ & Supplementary motor area & SMA & $(65,66)$ & Angular gyrus \\
$(21,22)$ & Olfactory cortex & OLF & $(67,68)$ & Precuneus \\
$(23,24)$ & Superior frontal gyrus, medial & SFGmed & $(69,70)$ & Paracentral lobule \\
$(25,26)$ & Superior frontal gyrus, medial orbital & ORBsupmed & $(71,72)$ & Caudate nucleus \\
$(27,28)$ & Gyrus rectus & REC & $(73,74)$ & Lenticular nucleus, putamen \\
$(29,30)$ & Insula & INS & $(75,76)$ & Lenticular nucleus, pallidium \\
$(31,32)$ & Anterior cingulate and paracingulate gyri & ACG & $(77,78)$ & Thalamus \\
$(33,34)$ & Median cingulate and paracingulate gyri & DCG & $(79,80)$ & Heschl gyrus \\
$(35,36)$ & Posterior cingulate gyrus & PCG & $(81,82)$ & Superior temporal gyrus \\
$(37,38)$ & Hippocampus & HIP & $(83,84)$ & Temporal pole: superior temporal gyrus \\
$(39,40)$ & Parahippocampal gyrus & PHG & $(85,86)$ & Middle temporal gyrus \\
$(41,42)$ & Amygdala & AMYG & $(87,88)$ & Temporal pole: middle temporal gyrus \\
$(43,44)$ & Calcarine fissure and surrounding cortex & CAL & $(89,90)$ & Inferior temporal gyrus \\
$(45,46)$ & Cuneus & CUN & PCUN \\
\hline
\end{tabular}

Note: The regions are listed according to a prior template obtained from an AAL atlas.

(WM) degeneration (Delbeuck et al., 2003; He et al., 2009). Advances in the development and application of diffusion tensor imaging (DTI) and graph theory methods allow for the investigation of topological patterns of human WM networks in vivo (Bullmore and Sporns, 2009; He and Evans, 2010; Stam, 2010; Bullmore and Bassett, 2011; Sporns, 2011). In healthy populations, WM networks have been mapped using deterministic (Hagmann et al., 2008; Gong et al., 2009a; Shu et al., 2009, 2011) or probabilistic tractography methods (Iturria-Medina et al., 2008; Gong et al., 2009b). These resultant networks exhibited nontrivial topological properties such as small worldness and network hubs. Moreover, this model has accelerated our understanding of aberrant structural connectivity in AD (Lo et al., 2010). Although both RGD and aMCI could be risk factors for developing $\mathrm{AD}$ at a later stage, no study has directly examined the topological organization of WM networks between RGD and aMCI.

Here, we used DTI tractography and graph theory approaches to investigate changes in the topological organization of the WM network in first-episode RGD and aMCI. We sought to determine whether WM networks would show (1) abnormal topological organization in patients with RGD and aMCI, and (2) similarities and differences in the patterns of deficits between the two patient groups.

\section{Materials and Methods}

Subjects

The present study recruited 103 elderly individuals (all of whom were Chinese Han and right-handed), including 35 RGD subjects (14 males and 21 females), 38 aMCI subjects (25 males and 13 females), and 30 healthy controls (16 males and 14 females), through normal community health screening, newspaper advertisement, and hospital outpatient service. A written informed consent was obtained from all of the participants, and the study was approved by the Research Ethics Committee of Affiliated ZhongDa Hospital, Southeast University. Some of these subjects were previously enrolled in our independent studies (Bai et al., 2009a; Yuan et al., 2010).
All first-episode RGD subjects (i.e., who were not depressed after the remission of the depressive symptoms) were interviewed in a semistructured interview included in the Structured Clinical Interview for Diagnostic and Statistical Manual of Mental Disorders, Fourth Edition (DSM-IV) Axis I Disorders (SCID-I), Clinician Version, and met the following inclusion criteria: (1) all subjects had previously met DSM-IV criteria for major depression disorder and had remitted for $>6$ months before enrollment, (2) these subjects had only one depressive episode with an age of onset was $>60$ years, (3) Hamilton Depression Rating Scale (HDRS) scores were $<7$ and Mini Mental State Exam score (MMSE) scores were $>24$, (4) duration of illness was $<5$ years and the medication-free period for all subjects was longer than 3 months before the assessment. Exclusion criteria were as follows: (1) another major psychiatric illness, including substance abuse or dependence; (2) primary neurological illness, including dementia or stroke; (3) medical illness impairing cognitive function; (4) history of electroconvulsive therapy; (5) T2-weighted MRI of all subjects showing major WM changes, infarction, or other lesions (two experienced radiologists executed the scans). The RGD subjects had a mean age of illness onset at $64.86( \pm 4.36)$ years. The mean duration of illness was $3.10( \pm 1.21)$ years. All patients had previously taken antidepressant medication: 25 patients, selective serotonin reuptake inhibitors, and 10 , serotonin-noradrenaline reuptake inhibitors.

All aMCI subjects met the diagnostic criteria proposed by Petersen et al. (1999) and others (Winblad et al., 2004): including (1) subjective memory impairment corroborated by subject and an informant; (2) objective memory performances documented by an Auditory Verbal Learning Test-delayed recall score that is $\leq 1.5 \mathrm{SD}$ of age-adjusted and education-adjusted norms (the cutoff was $\leq 4$ correct responses on 12 items for $\geq 8$ years of education); (3) normal general cognitive functioning evaluated by a MMSE of 24 or higher; (4) a Clinical Dementia Rating of 0.5 , with at least a 0.5 in the memory domain; (5) no or minimal impairment in activities of daily living; (6) absence of dementia, or not sufficient to meet the National Institute of Neurological and Communicative Disorders and Stroke and the Alzheimer's Disease and Related Disorders Association criteria for AD. Exclusion criteria were as follows: (1) a past history of known stroke (modified Hachinski score of $>4$ ), alcoholism, head injury, Parkinson's disease, epilepsy, major depression (excluded by Self-Rating Depression Scale), or other neurological or psychiatric illness (excluded by clinical assessment and case history); (2) 

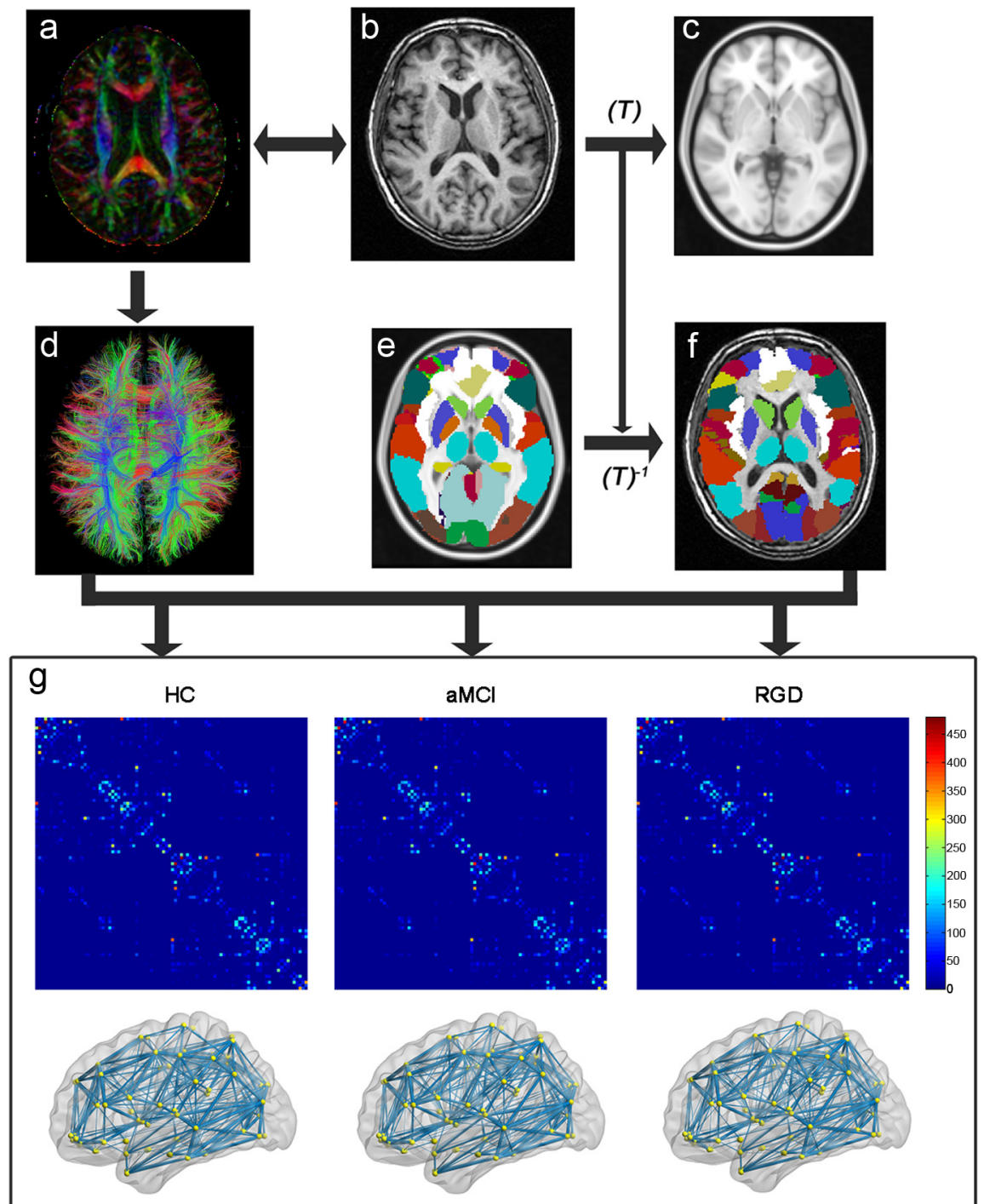

Figure 1. A flow chart for the construction of the WM structural network by DTI. (1) The rigid coregistration from the T1weighted structural MRI ( $\boldsymbol{b}$ ) to the DTI native space ( $\boldsymbol{a}$ ) (DTI color-coded map; red: left to right; green: anterior to posterior; blue: inferior to superior) for each subject. (2) The nonlinear registration from the resultant structural MRI to the ICBM152 T1 template in the MNI space (c), resulting in a nonlinear transformation (T). (3) The application of the inverse transformation $\left(T^{-1}\right)$ to the AAL template in the MNI space $(\boldsymbol{e})$, resulting in a subject-specific AAL mask in the DTI native space $(\boldsymbol{f})$. All of the registrations were implemented in the SPM8 package. (4) The reconstruction of all of the WM fibers (d) in the whole brain using DTI deterministic tractography in DTI-Studio. (5) The weighted networks of each subject were created by computing the fiber numbers (FNweighted) that connected each pair of brain regions. The matrices and 3D representations (lateral view) of the mean WM structural networks of each group are shown in the bottom panel $(\boldsymbol{g})$. The nodes and connections were mapped onto the cortical surfaces using the in-house BrainNet viewer software (www.nitrc.org/projects/bnv/). For details, see Materials and Methods. HC, Healthy controls.

major medical illness (e.g., cancer, anemia, and thyroid dysfunction); (3) severe visual or hearing loss; (4) T2-weighted MRI of all subjects showing major WM changes, infarction, or other lesions (two experienced radiologists executed the scans).

The control subjects were required to have a clinical dementia rating of 0 , an MMSE score of $\geq 26$, and a delayed recall score of $>4$ for those with $\geq 8$ years of education. These participants also met the aforementioned exclusion criteria for RGD and aMCI.

\section{MRI data acquisition}

The subjects were scanned using a 1.5 tesla scanner (General Electric Medical Systems) with a homogeneous birdcage head coil. Subjects lay supine with the head snugly fixed by a belt and foam pads to minimize head motion. Conventional axial fast relaxation fast spin echo sequence
T2-weighted anatomic MR images were obtained to rule out major WM changes, cerebral infarction, or other lesions: repetition time (TR), $3500 \mathrm{~ms}$; echo time (TE), $103 \mathrm{~ms}$; flip angle, $90^{\circ}$; acquisition matrix, $320 \times 192$; field of view $(\mathrm{FOV}), 240 \mathrm{~mm} \times 240 \mathrm{~mm}$; thickness, 6.0 $\mathrm{mm}$; gap, $0 \mathrm{~mm}$; number of excitations (NEX), 2. High-resolution T1-weighted axial images covering the whole brain were acquired using a $3 \mathrm{D}$ spoiled gradient echo sequence as follow: TR, 9.9 $\mathrm{ms}$; TE, $2.1 \mathrm{~ms}$; flip angle, $15^{\circ}$; acquisition matrix, $256 \times 192$; FOV, $240 \mathrm{~mm} \times 240 \mathrm{~mm}$; thickness, $2.0 \mathrm{~mm}$; gap, $0 \mathrm{~mm}$. Diffusion-weighted imaging was acquired with single-shot echo-planar imaging sequence in alignment with the anteriorposterior commissural plane. The diffusion sensitizing gradients were applied along 25 noncollinear directions $\left(b=1000 \mathrm{~s} / \mathrm{mm}^{2}\right)$, together with an acquisition without diffusion weighting $(b=0)$. Thirty contiguous axial slices were acquired with a slice thickness of $4 \mathrm{~mm}$ and no gap. The acquisition parameters were as follows: TR, 10,000 ms; TE, $81.2 \mathrm{~ms}$; flip angle, $90^{\circ}$; acquisition matrix, $128 \times 128$; FOV, $240 \times 240 \mathrm{~mm}$; NEX, 2.

\section{Data preprocessing}

The data preprocessing consisted of the following steps: eddy current and motion artifact correction of the DTI data (FSL; http://www.fmrib.ox. ac.uk/fsl), estimation of the diffusion tensor, calculation of the fractional anisotropy (FA), and diffusion tensor tractography. The eddy current distortions and motion artifacts in the DTI dataset were corrected for by applying an affine alignment of each diffusion-weighted image to the $b=$ 0 image. After this process, the diffusion tensor elements were estimated by solving the Stejskal and Tanner equation, and then, the reconstructed tensor matrix was diagonalized to obtain three eigenvalues $\left(\lambda_{1}, \lambda_{2}, \lambda_{3}\right)$ and eigenvectors. The FA of each voxel was also calculated. Diffusion tensor tractography was implemented using DTI-Studio software (H. Jiang and S. Mori, The Johns Hopkins University, Baltimore, MD) by using the "fiber assignment by continuous tracking" method (Mori et al., 1999). All of the tracts in the dataset were computed by seeding each voxel with an FA that was $>0.2$. The tractography was terminated if it turned an angle $>45^{\circ}$ or reached a voxel with an FA of $<0.2$ (Mori et al., 1999). The tractography was performed in each subject to generate three-dimensional curves that characterize neural fiber tract connectivity (Conturo et al., 1999; Basser et al., 2000), and it was performed using DTI-Studio. Tens of thousands of streamlines were generated to etch out all of the major WM tracts. To test the effects of the FA threshold of tractography on the results of the network analyses, we also performed whole-brain WM tractography with an FA threshold of 0.3 and constructed a WM network for each participant. Similar results of the network analyses were obtained (data not shown).

\section{Network construction}

The WM connectivity was modeled as a network comprising a total of 90 nodes (Table 1, Fig. 1). In this study, we defined all of the network nodes and edges using the following procedures.

Network node definition. The procedure used to define the nodes has been previously described (Gong et al., 2009a; Shu et al., 2009, 2011) and was performed in this study using SPM8 software (http://www.fil.ion. ucl.ac.uk/spm/software/spm8). Briefly, individual T1-weighted images 
Table 2. Demographic and neuropsychological data

\begin{tabular}{|c|c|c|c|c|c|}
\hline & Control $(n=30)$ & $\mathrm{aMCl}(n=38)$ & $\operatorname{RGD}(n=35)$ & Fvalue $\left(X^{2}\right)$ & $p$ value \\
\hline Age (years) & $71.3 \pm 4.4$ & $71.6 \pm 5.3$ & $67.9 \pm 4.5$ & 6.35 & $0.003^{*}$ \\
\hline Education (years) & $14.5 \pm 2.8$ & $13.5 \pm 3.2$ & $14.1 \pm 1.9$ & 1.29 & $0.28^{*}$ \\
\hline Gender (M/F) & $16 / 14$ & $25 / 13$ & $14 / 21$ & 4.87 & $0.088^{\#}$ \\
\hline MMSE & $28.43 \pm 1.25$ & $27.03 \pm 1.57$ & $28.54 \pm 2.19$ & 8.65 & $0.0003^{a, c}$ \\
\hline Auditory verbal memory test & $7.83 \pm 1.53$ & $3.61 \pm 1.53$ & $5.94 \pm 3.10$ & 31.68 & $<0.0001^{a, b, c}$ \\
\hline Auditory verbal memory test-delayed recall & $7.70 \pm 1.71$ & $2.61 \pm 1.26$ & $6.29 \pm 2.47$ & 68.84 & $<0.0001^{a, b, c}$ \\
\hline Rey-0sterrieth complex figure test & $34.53 \pm 1.80$ & $30.96 \pm 7.77$ & $30.03 \pm 8.85$ & 3.60 & $0.031^{a, b}$ \\
\hline Rey-0sterrieth complex figure test-delayed recall & $16.15 \pm 6.63$ & $10.37 \pm 6.80$ & $14.81 \pm 8.26$ & 6.06 & $0.0033^{a, c}$ \\
\hline Trail making test-A (seconds) & $70.93 \pm 28.70$ & $94.45 \pm 35.57$ & $115.83 \pm 83.34$ & 5.31 & $0.0065^{b}$ \\
\hline Trail making test- $B$ (seconds) & $133.50 \pm 40.00$ & $190.61 \pm 81.78$ & $218.49 \pm 155.91$ & 5.34 & $0.0062^{a, b}$ \\
\hline Symbol digit modalities test & $35.50 \pm 10.19$ & $25.66 \pm 9.75$ & $26.97 \pm 13.76$ & 7.08 & $0.0013^{a, b}$ \\
\hline Digit span test & $13.20 \pm 2.20$ & $11.97 \pm 1.99$ & $12.14 \pm 2.92$ & 2.46 & NS \\
\hline Clock drawing test & $9.07 \pm 1.05$ & $8.34 \pm 1.32$ & $8.31 \pm 2.19$ & 2.23 & NS \\
\hline HDRS & $0.89 \pm 1.03$ & - & $1.26 \pm 1.09$ & - & - \\
\hline
\end{tabular}

Values are represented as the mean \pm SD. For comparisons of demographics, ${ }^{*} p$ values were obtained using one-way ANOVA tests; ${ }^{*} p$ value for the gender distribution in the three groups was obtained using a $\chi^{2}$ test. The comparisons of neuorpsychological scores among the three groups (aMCI, RGD, and controls) were performed using a separate one-way ANOVA. Post hoc pairwise comparisons were then performed using $t$ test. $p<0.05$ was considered significant. NS, Not significant.

${ }^{a}$ Post hoc paired comparisons showed significant group differences between control versus aMCI.

${ }^{b}$ Post hoc paired comparisons showed significant group differences between control versus RGD.

'Post hoc paired comparisons showed significant group differences between aMCI versus RGD.

Table 3. Comparisons of the global network measures among the control, aMCI, and RGD groups

\begin{tabular}{|c|c|c|c|c|c|c|c|c|}
\hline & $S_{p}$ & $E_{\text {glob }}$ & $E_{\text {loc }}$ & $L_{p}$ & $C_{p}$ & $\lambda$ & $\gamma$ & $\sigma$ \\
\hline \multicolumn{9}{|c|}{ Low-resolution network (L-AAL) } \\
\hline Control & $579.6(14.48)$ & $0.60(0.009)$ & $0.94(0.011)$ & $1.67(0.029)$ & $0.36(0.002)$ & $1.17(0.008)$ & $4.00(0.088)$ & $3.43(0.069)$ \\
\hline $\mathrm{aMCl}$ & $512.6(13.20)$ & $0.57(0.008)$ & $0.91(0.010)$ & $1.77(0.026)$ & $0.36(0.002)$ & $1.18(0.007)$ & $4.17(0.080)$ & $3.54(0.062)$ \\
\hline Fvalue & 6.18 & 3.93 & 2.63 & 3.97 & 1.22 & 2.80 & 1.13 & 0.77 \\
\hline$p$ value & $0.003^{a, b}$ & $0.023^{a, b}$ & NS & $0.022^{a, b}$ & NS & NS & NS & NS \\
\hline \multicolumn{9}{|c|}{ High-resolution network (H-1024) } \\
\hline RGD & $84.17(2.00)$ & $0.32(0.005)$ & $0.77(0.008)$ & $3.18(0.056)$ & $0.32(0.002)$ & $1.37(0.009)$ & $24.76(0.38)$ & $18.06(0.21)$ \\
\hline Fvalue & 5.80 & 8.32 & 3.44 & 7.62 & 1.34 & 1.95 & 2.07 & 1.25 \\
\hline$p$ value & $0.004^{a, b}$ & $0.0005^{a, b}$ & $0.036^{a, b}$ & $0.0008^{a, b}$ & NS & NS & NS & NS \\
\hline
\end{tabular}

The FN-weighted WM networks for each participant were constructed using two parcellation methods (L-AAL and H-1024). The comparisons of the global network measures were performed among the three groups (aMCI, RGD, and controls) using univariate ANCOVAs. Post hoc pairwise comparisons were then performed using $t$ test. Values estimated the marginal means (SEs) of the global network measures after covarying out age, gender, and education effects. $p<0.05$ was considered significant. NS, Not significant.

${ }^{a}$ Post hoc paired comparisons showed significant group differences between control versus aMCl.

${ }^{b}$ Post hoc paired comparisons showed significant group differences between control versus RGD.

were coregistered to the b0 images in the DTI space. The transformed T1 images were then nonlinearly transformed into the ICBM152 T1 template in the MNI space. The inverse transformations were used to warp the automated anatomical labeling (AAL) atlas (Tzourio-Mazoyer et al., 2002) from the MNI space to the DTI native space. Of note, discrete labeling values were preserved using a nearest-neighbor interpolation method. Using this procedure, we obtained 90 cortical and subcortical regions ( 45 for each hemisphere) (Table 1), each representing a node of the network (Fig. 1).

Network edge definition. To define the network edges of the 90 regions, we selected a threshold value for the fiber bundles. Two regions were considered structurally connected if at least three fibers $(T=3)$ with two endpoints were located in these two regions (Shu et al., 2011). Such a threshold selection reduced the risk of false-positive connections due to noise or the limitations in the deterministic tractography and simultaneously ensured the size of the largest connected component (i.e., 90) that was observed in the networks across all of the controls (Shu et al., 2011). In this study, we also evaluated the effects of different thresholds on the network analysis by setting threshold values of the number of fiber bundles to range from 1 to 5 . We found that the threshold procedure did not significantly influence our results (see Results). If not indicated otherwise, we reported our results mainly based on a value of $T=3$. After defining the network edges, the weighted network analyses were performed. Specifically, we defined the fiber number (FN) of the connected fibers between two regions as the weights of the network edges. As a result, we constructed the FN-weighted WM network for each participant, which was represented by a symmetric $90 \times 90$ matrix (Fig. 1).

\section{Network analysis}

To characterize the topological organization of WM structural networks, several graph measures were considered here, as follows: network strength, global efficiency, local efficiency, shortest path length, clustering coefficient, and small worldness (Rubinov and Sporns, 2010). For a recent review on the uses and interpretations of these network measures, see Rubinov and Sporns (2010) and the following descriptions.

Network strength. For a network (graph) $G$ with $N$ nodes and $K$ edges, we calculated the strength of $G$ as follows:

$$
S_{p}(G)=\frac{1}{N} \sum_{i \in G} S(i)
$$

where $S(i)$ is the sum of the edge weights $w_{i j}\left(w_{i j}\right.$ are the FN values for the weighted networks) linking to node $i$. The strength of a network is the average of the strength across all of the nodes in the network. To control for the effects of the different number of total fiber on the network topological differences, the connectivity matrix of each subject was first normalized by the network strength (the total number of interconnect- 

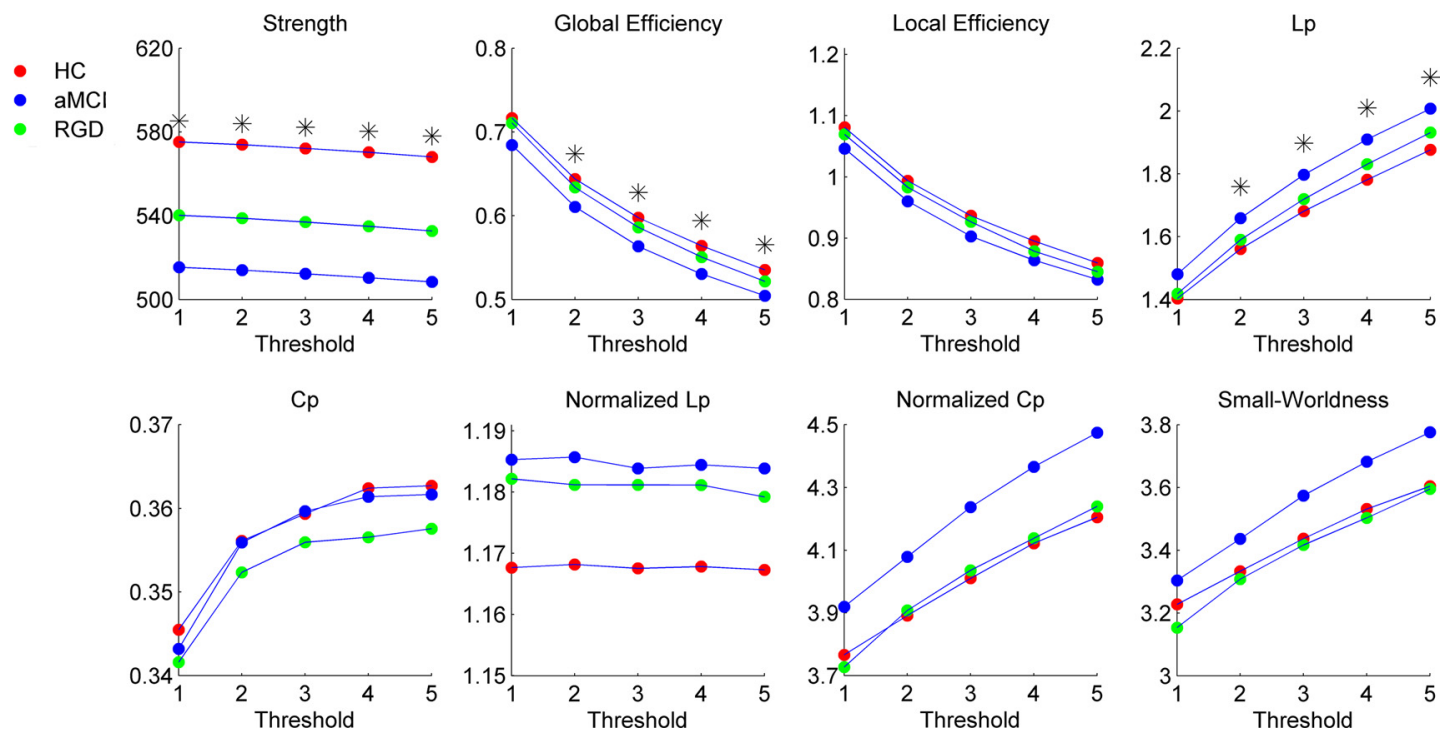

Figure 2. Global measures of WM structural networks were quantified in the control, aMCl, and RGD patients. The threshold (horizontal axis) determined the minimum number of streamlines that needed to interconnect a pair of nodes for a connection to be assumed. The data points are marked with an asterisk to indicate a significant difference ( $p<0.05$ ) among the three groups. Note that $T=n(1,2,3,4,5)$ indicates at least $n$ or more streamlines must be present for a link to be drawn. Significant group effects in the network strength, global efficiency, and absolute path length were observed for most of the thresholds. Note that the FN-weighted WM network for each participant was constructed using the AAL template. HC, Healthy controls.

ing fibers between regions) before the calculation of the following network properties.

Small-world properties. Small-world network parameters (clustering coefficient, $C_{p}$, and shortest path length, $L_{p}$ ) were originally proposed by Watts and Strogatz (1998).

In this study, we investigated the small-world properties of the weighted brain networks. The clustering coefficient of a node $i, C(i)$, which was defined as the likelihood of whether the neighborhoods were connected with each other or not, was computed as follows:

$$
C(i)=\frac{2}{k_{i}\left(k_{i}-1\right)} \sum_{j, k}\left(\bar{w}_{i j} \bar{w}_{j k} \bar{w}_{k i}\right)^{1 / 3},
$$

where $k_{i}$ is the degree of node $i$ and $\bar{w}$ is the weight, which is scaled by the mean of all weights to control each participant's cost at the same level. The clustering coefficient is zero $[C(i)=0]$ if the nodes are isolated or have just one connection (i.e., $k_{i}=0$ or $k_{i}=1$ ). The clustering coefficient, $C_{p}$, of a network is the average of the clustering coefficient over all nodes and indicates the extent of the local interconnectivity or cliquishness in a network (Watts and Strogatz, 1998).

The path length between any pair of nodes (e.g., node $i$ and node $j$ ) is defined as the sum of the edge lengths along this path. For weighted networks, the length of each edge was assigned by computing the reciprocal of the edge weight, $1 / w_{i j}$. The shortest path length, $L_{i j}$, is defined as the length of the path for node $i$ and node $j$ with the shortest length. The shortest path length of a network was computed as follows:

$$
L_{p}(G)=\frac{1}{N(N-1)} \sum_{i \neq j \in G} L_{i j}
$$

where $N$ is the number of nodes in the network. The $L_{p}$ of a network quantifies the ability for information to propagate in parallel.

To examine the small-world properties, the clustering coefficient, $C_{p}$, and the shortest path length, $L_{p}$, of the brain networks were compared with those of random networks. In this study, we generated 100 matched random networks, which had the same number of nodes, edges, and degree distribution as the real networks (Maslov and Sneppen, 2002). Of note, we retained the weight of each edge during the randomization procedure such that the weight distribution of the network was preserved. Furthermore, we computed the normalized shortest path length $(\lambda), \lambda=L_{p}^{\text {real }} / L_{p}^{\text {rand }}$, and the normalized clustering coefficient $(\gamma), \gamma=C_{p}^{\text {real }} / C_{p}^{\text {rand }}$, where $L_{p}^{\text {rand }}$ and $C_{p}^{\text {rand }}$ are the mean clustering coefficient and the mean shortest path length of 100 matched random networks, respectively. Importantly, two parameters correct the differences in the edge number and degree distribution of the networks across individuals. A real network would be considered small-world if $\gamma>1$ and $\lambda \approx 1$ (Watts and Strogatz, 1998). Thus, a small-world network not only has a higher local interconnectivity but also has an approximately equivalent shortest path length compared with random networks. These two measurements can be summarized into a simple quantitative metric, small-worldness, $\sigma=$ $\gamma / \lambda$, which is typically $>1$ for small-world networks (Humphries et al., 2005).

Network efficiency. The global efficiency of $G$ measures the global efficiency of the parallel information transfer in the network (Latora and Marchiori, 2001), which can be computed as follows:

$$
E_{\text {glob }}(G)=\frac{1}{N(N-1)} \sum_{i \neq j \in G} \frac{1}{L_{i j}},
$$

where $L_{i j}$ is the shortest path length between node $i$ and node $j$ in $G$.

The local efficiency of $G$ reveals how much the network is fault tolerant and shows how efficient the communication is among the first neighbors of the node $i$ when it is removed. The local efficiency of a graph is defined as follows:

$$
E_{\mathrm{loc}}(G)=\frac{1}{N} \sum_{i \in G} E_{\mathrm{glob}}\left(G_{i}\right)
$$

where $G_{i}$ denotes the subgraph composed of the nearest neighbors of node $i$.

Regional nodal characteristics. To determine the nodal (regional) characteristics of the WM networks, we computed the regional efficiency, $E_{\text {nodal }}(i)$ (Achard and Bullmore, 2007), as follows:

$$
E_{\text {nodal }}(i)=\frac{1}{N-1} \sum_{i \neq j \in G} \frac{1}{L_{i j}},
$$

where $L_{i j}$ is the shortest path length between node $i$ and node $j$ in $G$. $E_{\text {nodal }}(i)$ measures the average shortest path length between a given node $i$ and all of the other nodes in the network. The node $i$ is a brain hub if $E_{\text {nodal }}(i)$ is at least $1 \mathrm{SD}$ greater than the average nodal efficiency of the network [i.e., $E_{\text {nodal }}(i)>$ mean $+\mathrm{SD}$ ].

Network connectivity characteristics. To further localize specific pairs of brain regions in which the structural connectivity was altered in patients, we used a network-based statistic (NBS) approach (Zalesky et al., 
2010b). Briefly, we identified regional pairs, which show between-group differences in structural connectivity, and used the NBS method to localize connected networks that show significant changes in both the aMCI and RGD patients.

High-resolution brain network analysis It has been previously shown that brain graph metrics are dependent on the resolution of the network (i.e., network size) (van den Heuvel et al., 2008; Wang et al., 2009; Zalesky et al., 2010a). Recent studies have suggested the use of higher-resolution networks, of up to 1000 smaller parcels, instead of using a coarse parcellation scheme of 90 brain regions (Hagmann et al., 2008; van den Heuvel et al., 2008). Therefore, we further subdivided the AAL template into 1024 ROIs with equal size [i.e., high-resolution $(\mathrm{H}-$ 1024)] (Zalesky et al., 2010a). Then, we performed a high-resolution network analysis to examine the topological alterations in aMCI and RGD patients. Similar to the lowresolution AAL (L-AAL) networks, the number of interconnecting fibers was defined as the weight of the network edge, which results in a symmetric $1024 \times 1024$ matrix for each participant.

\section{Statistical analysis}

To test the group differences in age, years of education, and neuropsychological scores, we analyzed the data with separate one-way ANOVAs. Post hoc pairwise comparisons were then performed using $t$ tests. The gender data were analyzed using a $\chi^{2}$ test.

To determine the group differences in global network measures and regional nodal characteristics, comparisons were performed among the three groups using univariate ANCOVAs. Post hoc pairwise comparisons were then performed using a general linear model. The effects of age, gender, and years of education were adjusted for all of these analyses. A value of $p<0.05$ was considered significant.

To determine the significance levels of altered connectivity networks in the NBS analysis, we first detected the significant nonzero connections within each group by performing a nonparametric onetailed sign test. For each pair of brain regions, the sign test was performed with the null hypothesis that there is no existing connection, that is, "fiber bundle number $=0$." The Bonferroni method was then used to correct for multiple comparisons (i.e., $90 \times 89 / 2=4005$ pairs of regions) at $p<0.05$. Next, the nonzero connections within either the patient or control groups were detected and combined into a connection mask. The NBS approach was then conducted within the connection mask, where a primary threshold $(p=0.05)$ was first applied to a $t$ statistic (two-sample one-tailed $t$ tests). This $t$ statistic was computed for each link to define a set of suprathreshold links among which any connected components and their size (number of links) could then be determined. To estimate the significance for each component, the null distribution of the connected component size was empirically derived using a nonparametric permutation approach (10,000 permutations). For each permutation, all of the subjects were randomly reallocated into two groups, and the $t$ statistic was computed independently for each link. Next, the threshold ( $p=$ 0.05 ) was used to generate suprathreshold links among which the maximal connected component size was recorded. Finally, for a connected component of size $M$ that was found in the right grouping of Table 1.

\section{$\mathrm{HC}$}

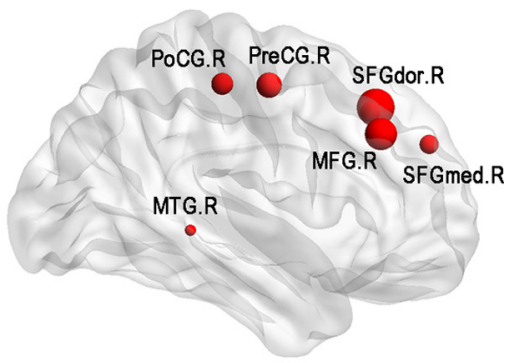

$\mathrm{aMCl}$

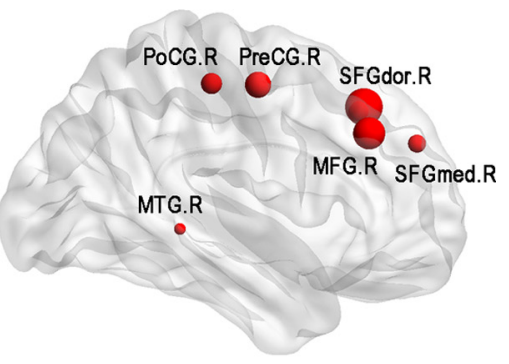

RGD
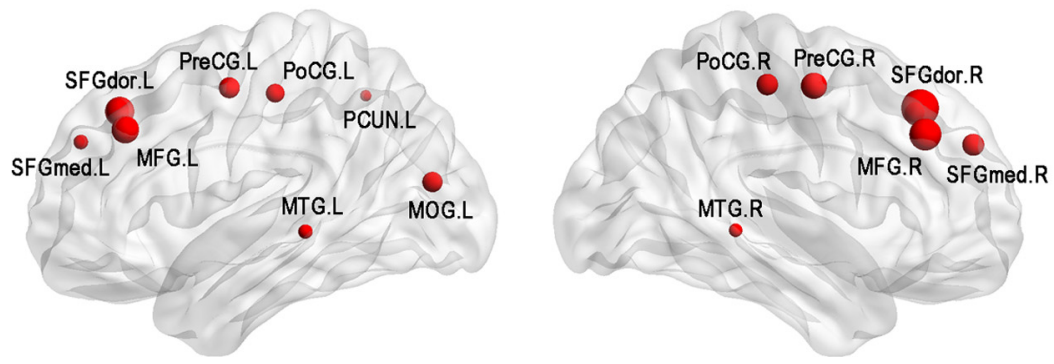

Figure 3. Three-dimensional representations of the hub distributions in the control, aMCl, and RGD groups. The hub nodes are shown in red with node sizes that represent their nodal efficiency values. The regions were mapped onto the cortical surface at a network for each participant was constructed using the AAL template. HC, Healthy controls. For the abbreviations of the nodes, see

controls and patients, the corrected $p$ value was determined by calculating the proportion of the 10,000 permutations for which the maximal connected component was larger than $M$. Of note, the effects of age, gender, and years of education were removed by a regression analysis performed before the statistical analysis of connections. For a detailed description, see the study by Zalesky et al. (2010b).

\section{Results}

\section{Neuropsychological test results}

The demographic data are shown in Table 2. Although the education and gender distribution were not different between the three groups $(p>0.05)$, one-way ANOVAs indicated that the three groups were not matched for age $(p<0.05)$ (it should be noted that the age effect was removed in all of the following network analyses). The neuropsychological characterizations for each group are presented in Table 2. The ANOVAs showed significant group effects in most of the test scores. Specifically, aMCI patients showed greater deficits in episodic memory (i.e., auditory verbal memory test-delayed recall and Rey-Osterrieth complex figure test-delayed recall) than RGD patients. In contrast, a tendency toward a greater alteration in executive function was observed in the RGD patients compared with the aMCI patients (i.e., trail-making tests, $\mathrm{A}$ and $\mathrm{B}$ ). 
Table 4. Hub Regions of WM networks in the control, aMCl, and RGD groups

\begin{tabular}{|c|c|c|c|c|}
\hline & Region & Class & $E_{i} /$ mean & $K_{i}$ \\
\hline \multirow[t]{14}{*}{ Control } & SFGdor.R & Association & 1.66 & 32.35 \\
\hline & SFGdor.L & Association & 1.60 & 26.21 \\
\hline & MFG.R & Association & 1.58 & 21.38 \\
\hline & MFG.L & Association & 1.58 & 21.44 \\
\hline & PreCG.R & Primary & 1.49 & 23.44 \\
\hline & PreCG.L & Primary & 1.48 & 21.69 \\
\hline & PoCG.L & Primary & 1.45 & 21.61 \\
\hline & PoCG.R & Primary & 1.45 & 22.33 \\
\hline & SFGmed.L & Association & 1.43 & 16.81 \\
\hline & SFGmed.R & Association & 1.41 & 14.77 \\
\hline & MOG.L & Association & 1.41 & 26.83 \\
\hline & MTG.L & Association & 1.33 & 21.65 \\
\hline & SOG.L & Association & 1.31 & 18.16 \\
\hline & MTG.R & Association & 1.31 & 20.77 \\
\hline \multirow[t]{14}{*}{$\mathrm{aMCl}$} & SFGdor.R & Association & 1.60 & 28.82 \\
\hline & MFG.R & Association & 1.54 & 20.74 \\
\hline & SFGdor.L & Association & 1.52 & 23.05 \\
\hline & MFG.L & Association & 1.50 & 19.16 \\
\hline & PreCG.R & Primary & 1.47 & 21.53 \\
\hline & PreCG.L & Primary & 1.46 & 20.39 \\
\hline & MOG.L & Association & 1.44 & 24.80 \\
\hline & PoCG.L & Primary & 1.44 & 21.08 \\
\hline & PoCG.R & Primary & 1.41 & 20.31 \\
\hline & SFGmed.R & Association & 1.38 & 14.37 \\
\hline & SFGmed.L & Association & 1.37 & 14.78 \\
\hline & SOG.L & Association & 1.34 & 17.06 \\
\hline & MTG.L & Association & 1.33 & 20.28 \\
\hline & MTG.R & Association & 1.31 & 18.58 \\
\hline \multirow[t]{14}{*}{ RGD } & SFGdor.R & Association & 1.65 & 32.74 \\
\hline & MFG.R & Association & 1.58 & 22.01 \\
\hline & SFGdor.L & Association & 1.54 & 25.04 \\
\hline & MFG.L & Association & 1.52 & 20.23 \\
\hline & PreCG.R & Primary & 1.50 & 23.35 \\
\hline & SFGmed.R & Association & 1.45 & 16.36 \\
\hline & PoCG.R & Primary & 1.44 & 22.13 \\
\hline & PreCG.L & Primary & 1.43 & 20.74 \\
\hline & MOG.L & Association & 1.43 & 25.33 \\
\hline & PoCG.L & Primary & 1.41 & 21.12 \\
\hline & SFGmed.L & Association & 1.35 & 15.06 \\
\hline & MTG.L & Association & 1.35 & 22.30 \\
\hline & MTG.R & Association & 1.34 & 20.58 \\
\hline & PCUN.L & Association & 1.31 & 22.62 \\
\hline
\end{tabular}

The FN-weighted WM network for each participant was constructed using an AAL template. The hub regions were identified if $E_{\text {nodal }}(i)$ was at least 1 SD greater than the mean nodal efficiency of the network $\left[\right.$ i.e., $E_{\text {nodal }}(i)>$ mean $\pm S D$ ]. The hubs were then sorted by the mean normalized nodal efficiency (divided by the mean of all nodes) in each group. The cortical regions were classified as primary, association, and paralimbic.

\section{Global topology of the WM structural networks}

Both of the patients and control subjects showed a small-world organization of the WM networks, as expressed by $\gamma>1$ and $\lambda \approx$ 1 (Table 3, Fig. 2). Among the three groups, ANCOVAs on the global network properties showed significant group effects in network strength, global efficiency, and absolute shortest path length (Table 3). In addition, post hoc comparisons showed significantly reduced strength, decreased global efficiency, and increased absolute path length in both the RGD and aMCI patients relative to the controls. However, no differences (all $p>0.5$ ) were found between the RGD and aMCI patients in these parameters (Table 3).
Table 5. Brain regions with significant group effects in the nodal efficiency among the control, aMCI, and RGD groups

\begin{tabular}{llllll}
\hline & & & \multicolumn{2}{l}{$T$ value ( $p$ value) of post hoc test } \\
\cline { 4 - 6 } Regions & Category & $\begin{array}{l}\text { Fvalue ( } p \text { value) } \\
\text { of ANCOVA }\end{array}$ & $\begin{array}{l}\text { Control versus } \\
\text { aMCl }\end{array}$ & $\begin{array}{l}\text { Control versus } \\
\text { RGD }\end{array}$ & $\begin{array}{l}\text { aMCl versus } \\
\text { RGD }\end{array}$ \\
\hline SFGmed.L & Frontal & $6.18(0.003)$ & $-2.04(0.045)$ & $-3.27(0.002)$ & NS \\
SFGdor.L & Frontal & $5.26(0.007)$ & $-2.77(0.007)$ & $-2.78(0.007)$ & NS \\
MFG.L & Frontal & $4.85(0.010)$ & $-2.77(0.007)$ & $-2.39(0.020)$ & NS \\
ACG.L & Paralimbic & $4.26(0.017)$ & $-2.21(0.031)$ & $-2.82(0.006)$ & NS \\
SFGdor.R & Frontal & $3.42(0.037)$ & $-2.48(0.016)$ & $-2.08(0.042)$ & NS \\
ANG.L & Parietal & $3.38(0.038)$ & $-2.55(0.013)$ & NS & NS \\
IFGtriang.L & Frontal & $3.38(0.038)$ & $-3.01(0.004)$ & NS & NS \\
PCG.R & Paralimbic & $3.37(0.039)$ & NS & NS & $2.30(0.025)$ \\
PreCG.L & Frontal & $3.36(0.039)$ & $-2.17(0.034)$ & $-2.29(0.025)$ & NS \\
MFG.R & Frontal & $3.33(0.040)$ & $-2.64(0.010)$ & NS & NS \\
ANG.R & Parietal & $3.18(0.046)$ & $-2.27(0.026)$ & NS & NS \\
\hline
\end{tabular}

The FN-weighted WM network for each participant was constructed using an AAL template. The comparisons of nodal efficiency were performed among the three groups (aMCI, RGD, and controls) using univariate ANCOVAs. Post hoc pairwise comparisons were then performed using $t$ test. The age, gender, and education effects were removed in all of these analyses. For ANCOVAs of the 90 brain regions, $p<0.05$ (uncorrected) was considered significant. For post hoc tests, $p<0.05$ was considered significant. NS, Not significant. For $T$ values, each negative column represents aMCI < control, RGD < control, and $\mathrm{RGD}<\mathrm{aMCl}$, respectively.

\section{Node-based analysis}

Identification of network hubs

The hub nodes with a size of $E_{\text {nodal }}(i)$ on the cortical surfaces are shown in Figure 4. For each group, we identified 14 hub regions, including 10 association cortical regions and 4 primary cortical regions (Fig. 3, Table 4). In particular, 13 of the hub regions were the same for all of the groups, including the bilateral dorsal superior frontal gyrus (SFGdor), bilateral middle frontal gyrus (MFG), bilateral precentral gyrus (PreCG), bilateral postcentral gyrus (PoCG), bilateral superior frontal gyrus, medial (SFGmed), left middle occipital gyrus (MOG), and bilateral middle temporal gyrus (MTG). One hub region, the left superior occipital gyrus (SOG), was identified as a hub in the control and aMCI groups, but not in the RGD group. In addition, the left precuneus (PCUN) was identified as a hub in the RGD group, but not in the other two groups. Moreover, the hubs identified for all of the groups were predominantly found in regions of the association cortices. These results were approximately consistent with those obtained from previous studies (He et al., 2007; Hagmann et al., 2008; Iturria-Medina et al., 2008; Gong et al., 2009a; Shu et al., 2009, 2011).

Between-group differences in regional efficiency

Following the discovery of a disrupted global network organization, we further localized the regions with altered nodal efficiency in the patients (Table 5, Fig. 4). Among the three groups, we found that the regions with significant group effects were mainly distributed in the frontal and parietal cortices, including seven frontal regions [left SFGmed, bilateral SFGdor, bilateral MFG, left triangular part of the inferior frontal gyrus (IFGtriang), left anterior cingulate gyrus (ACG)] and four parietal regions [right posterior cingulate gyrus (PCG), left PreCG, and bilateral angular gyrus (ANG)]. Post hoc tests showed that most of these regions (10 of 11) had a reduced efficiency in aMCI patients compared with controls. In six of these regions, including the left SFGmed, bilateral SFGdor, left MFG, left ACG, and left PreCG, reduced efficiency was observed in the RGD patients compared with controls. In particular, decreased regional efficiency of the frontal structures (five regions) in the WM networks were common deficits between the RGD and aMCI. Only one region (i.e., right PCG) showed significant group differences between the RGD and aMCI patients, with a higher nodal efficiency observed in the 

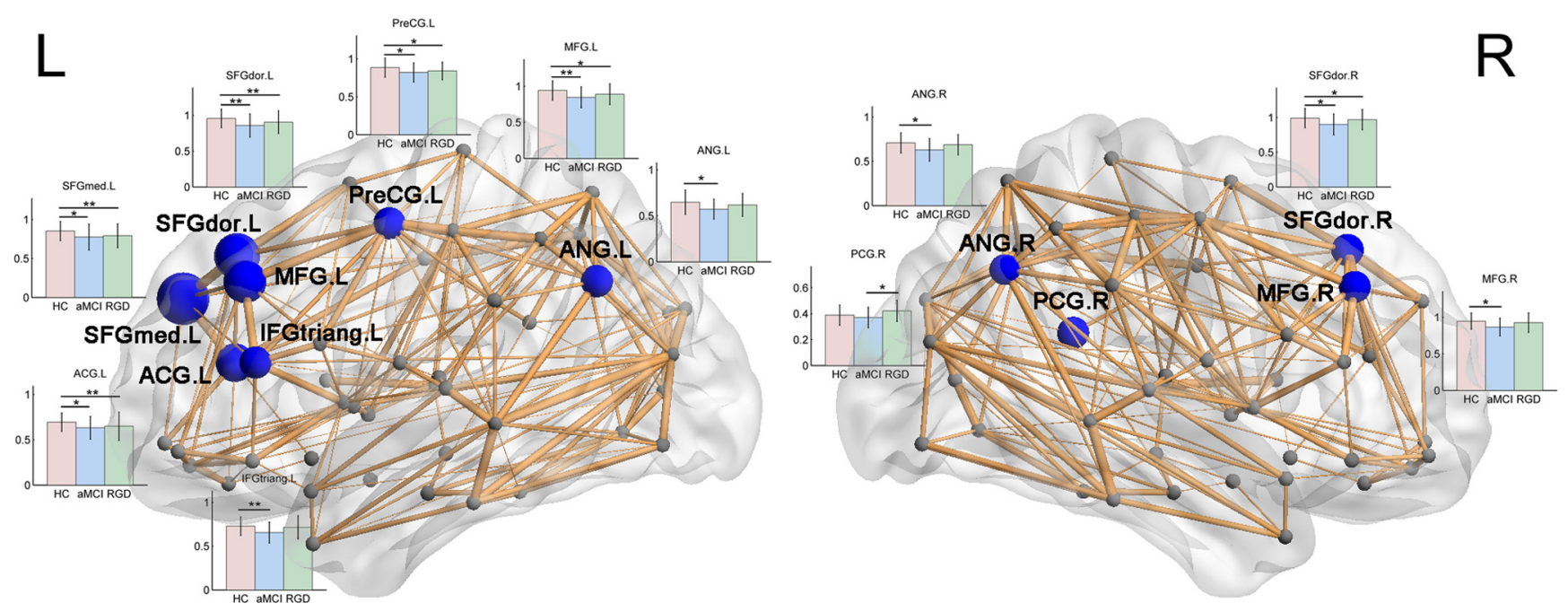

Figure 4. The distribution of brain regions with significant group effects in the nodal efficiency among the three groups at $p<0.05$ (uncorrected). The node sizes indicate the significance of between-group differences in the regional efficiency. For each node, the bar and error bar represent the mean value and SD, respectively, of the nodal efficiency in each group. Post hoc tests showed that most of these regions (10 of 11) have a reduced efficiency in the aMCl patients versus the controls. Six of these regions, including the left SFGmed, bilateral SFGdor, left MFG, left ACG, and left PreCG showed a reduced efficiency in the RGD patients compared with the controls. Only one region (right PCG) showed significant group differences between the RGD and aMCI patients, with a higher efficiency in RGD than in aMCl. A single asterisk (*) represents a significant group difference at $p<0.05$; a double asterisk $\left(^{* *}\right)$ represents a significant group difference at $p<0.01$. The network shown here was constructed by averaging the anatomical connection matrices of all healthy controls with a threshold of a sparsity of $10 \%$. Note that the FN-weighted WM network for each participant was constructed using the AAL template. The nodes and connections were mapped onto the cortical surfaces using in-house BrainNet viewer software (www.nitrc.org/projects/bnv/). HC, Healthy controls. For the abbreviations of the nodes, see Table 1.

RGD than the aMCI patients. However, this efficiency was no different from controls in either patient group.

\section{Connectivity-based analysis}

We used the NBS method to identify the disrupted connected components in patients (Fig. 5). For the aMCI group, a single connected network with 33 nodes and 35 edges was altered ( $p=$ 0.002 , corrected). The involved nodal regions mainly included the bilateral frontal [PreCG, SFGdor, SFGmed, MFG, IFGtriang, opercular part of inferior frontal gyrus (IFGoperc), and the supplementary motor area (SMA)], temporal [superior temporal gyrus (STG), MTG, and inferior temporal gyrus (ITG)] and paralimbic [orbital part of superior frontal gyrus (ORBsup), orbital part of middle frontal gyrus (ORBmid), medial orbital part of superior frontal gyrus (ORBsupmed), insula (INS), ACG, median cingulate and paracingulate gyri (DCG), olfactory cortex (OLF), gyrus rectus (REC), temporal pole of superior temporal gyrus (TPOsup), and temporal pole of the middle temporal gyrus (TPOmid)] regions (Fig. 5A). There were also two occipital regions [MOG and inferior occipital gyrus (IOG)], a parietal region (ANG), and a subcortical structure [amygdala (AMYG)] (Fig. $5 A)$. For the RGD group, a single connected network consisting of 18 nodes and 19 edges in the right hemisphere was altered ( $p=$ 0.015 , corrected). The nodes were primarily composed of several temporal (STG, MTG, and ITG), occipital [SOG, MOG, IOG, and fusiform gyrus (FFG)], paralimbic [ORBsup, INS, parahippocampal gyrus (PHG), TPOsup, and TPOmid] and subcortical [hippocampus (HIP), caudate nucleus (CAU), putamen (PUT), pallidium (PAL), and thalamus (THA)] regions (Fig. 5B). Importantly, all of the connections exhibited decreased values in the patients compared with the controls. No significant differences were found with respect to connected components between aMCI and RGD patients.

\section{High-resolution structural brain networks}

In addition to the L-AAL network analysis, an H-1024 network analysis was also performed. First, both the patients and control subjects showed a small-world topology of the high-resolution WM networks (Table 3). Among the three groups, significant group effects in network strength, global and local efficiencies, and the absolute shortest path length were observed. Post hoc comparisons showed significantly reduced strength, decreased global and local efficiencies, and increased absolute path length in both the RGD and aMCI patients compared with the controls. However, there were no differences (all $p>0.5$ ) found between the RGD and aMCI patients in these parameters. These results were comparable with those of the L-AAL network analyses. Second, the hub distributions of each group are shown in Figure $6 \mathrm{~A}$. We found that the hub distributions were also approximately similar to those obtained from the L-AAL network analyses, which were mainly distributed in the association cortices. Third, we identified the nodes with reduced efficiency in the patients $(p<0.005$, uncorrected) (Fig. $6 B)$, which were mainly distributed in the frontal brain regions. We determined that most of the nodes (36 of 42) exhibited a reduced efficiency in both the aMCI and RGD patients relative to the controls. Between the aMCI and RGD groups, three nodes in the prefrontal cortex showed a reduced efficiency ( $p<0.05$, uncorrected) in the RGD patients compared with the aMCI patients. In addition, one node in the left inferior frontal gyrus showed a reduced efficiency $(p<0.01$, uncorrected) in the aMCI patients than in the RGD patients.

\section{Discussion}

Using DTI and graph theory methods, we showed the topological alterations of WM networks in RGD and aMCI. The three main findings are as follows: (1) the organization of the WM network in these patients was significantly disrupted, as indicated by reduced network strength and global efficiency; (2) decreased regional and connectivity characteristics of primarily frontal structures were deficits common between the two patient groups; and (3) different regional/connectivity characteristics of the PCG and several prefrontal regions were observed between the RGD and aMCI groups. These findings extend our understand- 
A

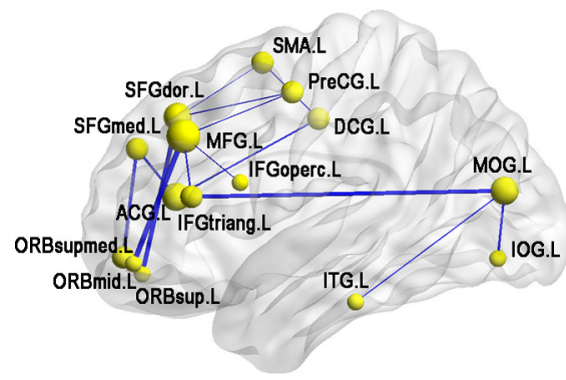

$\mathrm{aMCl}<\mathrm{HC}$
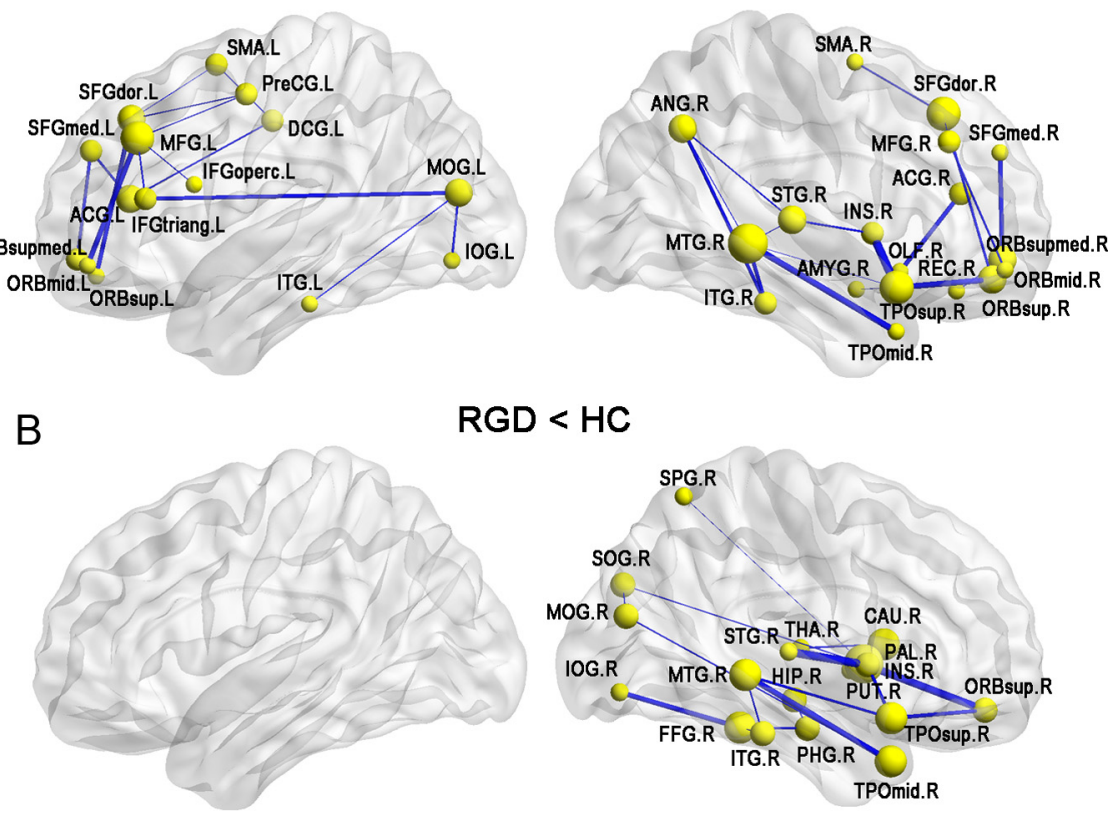

RGD $<$ HC

Figure 5. The connected networks showing decreased structural connections in the aMCI and RGD patients compared with the controls. $\boldsymbol{A}$, The regional pairs showing decreased connections in the aMCI patients. These connections formed a single connected network with 33 nodes and 35 connections ( $p=0.002$, corrected). $\boldsymbol{B}$, The regional pairs showing decreased connections in the RGD patients. These connections formed a single connected network with 18 nodes and 19 edges in right hemisphere $(p=0.015$, corrected). Between the aMCl and RGD patients, no connected components with significant difference were found. Note that the FN-weighted WM network for each participant was constructed using the AAL template. The nodes and connections were mapped onto the cortical surfaces using in-house BrainNet viewer software (www.nitrc.org/projects/bnv/).

ing of the neurophysiologic mechanisms involved in these two diseases from a network perspective.

\section{Disrupted global topological organization in the WM networks}

WM networks in the RGD, aMCI, and controls showed smallworld properties (i.e., high global and local efficiencies), which were consistent with previous diffusion MRI-based WM network studies performed in healthy adults (Hagmann et al., 2008; Iturria-Medina et al., 2008; Gong et al., 2009a,b; Shu et al., 2009, 2011; Lo et al., 2010). However, several topological properties, including network strength, global efficiency, and absolute path length, were significantly altered in the RGD and aMCI groups compared with controls. These findings were consistent with previous neuroimaging studies using graph analysis to study depression (Leistedt et al., 2009) and AD (Stam et al., 2007; He et al., 2008; Supekar et al., 2008; Lo et al., 2010). Lower network strength was related to the sparse connectivity of the brain networks, which suggests a reduced WM integrity in the RGD and aMCI patients. The global efficiency reflects the information transfer between remote regions and is mainly associated with long-range connections. Decreases in global efficiencies reflect a disrupted global integration of the WM networks in these patients, which could be due to disconnections between brain regions. In addition, short path lengths ensure interregional effective integrity or prompt transfer of information in brain networks, which constitutes the basis of cognitive processes (Sporns and Zwi, 2004). Thus, the disease-related increases in the absolute path length may be attributable to the degeneration of fiber bundles used for information transmission. Consistent with previous studies associated with WM disconnections in the brain of RGD (Yuan et al., 2010) and aMCI (Bai et al., 2009a), this study is the first to report topologically convergent and divergent patterns of the whole-brain WM networks between these patients.

\section{Common deficits of regional/ connection characteristics in the WM networks}

The common deficits of regional alterations in the RGD and aMCI networks were associated with decreased nodal efficiency, predominately located in the frontal lobe. This finding was similar to that of a recent WM network study of $\mathrm{AD}$, showing disconnections of the WM in these regions (Lo et al., 2010). Moreover, the abnormal frontal lobe has been reported in previous WM integrity studies of RGD (Alexopoulos et al., 2008; Yuan et al., 2010) and aMCI patients (Bai et al., 2009a; Zhuang et al., 2010; Sexton et al., 2011). Interestingly, our previous fMRI studies associated partly with the frontal regions indicated increased functional connectivity in RGD (Yuan et al., 2008b; Zhang et al., 2011) and aMCI patients (Bai et al., 2009b). We assumed that the decreases observed in the anatomical connectivity were likely to be associated with the compensation in functional connectivity. Interestingly, the altered patterns of the frontal regions appear to be similar in these two groups of patients. These frontal regions are primary sites that are the earliest affected by $\beta$-amyloid protein accumulation in the development of $\mathrm{AD}$ (Jack et al., 2008). The RGD subjects were found to have similar regional characteristics of frontal regions comparable with aMCI, providing additional support to the theory that RGD may be the prodromal manifestation of AD. Furthermore, the common deficits of decreased connections were mainly distributed between the frontal, temporal paralimbic regions and insula. Although there is a growing amount of evidence that supports the hypothesis of $\mathrm{AD}$ as a disconnection syndrome (Delbeuck et al., 2003; He et al., 2009), this study contributes toward the understanding of RGD and $\mathrm{AMCI}$ as a high risk for later developing AD.

\section{Disease-related distinctions of regional/connectivity characteristics in the WM networks}

A novel finding in this study was the identification of an increased tendency of nodal efficiency of the PCG in the L-AAL WM networks in the RGD compared with the aMCI patients. The PCG was initially thought to be one of the neural bases that mediate both emotional and memory functions (Goveas et al., 2011; Xie et al., 2011). Moreover, Goveas et al. (2011) found that a greater depressive symptom severity was associated with an increased function of the PCG in these interactions. However, the depressive symptoms were controlled with a HDRS score of $\leq 7$ after treatment in the present RGD subjects. A neuropathological study (Rapp et al., 2006) previously demonstrated that AD patients with a lifetime history of depression, including the diagnosis of current and past depressive disorder, showed higher levels of both plaque and tangles in the PCG than AD patients without depression. Our present findings are not fully consistent with this 
report because a greater pathology in the PCG in the aMCI than the RGD patients would best explain the differences observed between the two groups. However, we cannot exclude the possibility that the antidepressant medication of the RGD patients may be a reason for the high regional efficiency. Importantly, there was no significant disrupted nodal efficiency of the PCG in the WM networks between the patients and the controls, although previous DTI studies related to RGD (Yuan et al., 2010) and aMCI (Bai et al., 2009a) found altered regional integrity in this structure. This implied that the homeostasis (i.e., nodal efficiency of PCG) of complex networks allowing for the segregation and integration of information processing seemed to be more solid compared with regional changes. Another possible explanation of the increased nodal efficiency in RGD was that there was indisputable evidence of the early functional and structural impairments of PCG in aMCI (Fennema-Notestine et al., 2009; BeasonHeld, 2011) and its assumed role in memory processing in connection to hippocampus (Kenny et al., 2011), whereas the predominance of cognitive impairments in aMCI is associated with memory function (Petersen et al., 1999). In addition, our high-resolution structural brain networks showed sporadic prefrontal regions that were associated with a discrepant nodal efficiency in the WM networks in the RGD compared with aMCI patients. The prefrontal lobe system usually plays a vital role in mood regulation and cognitive function (Milak et al., 2005; Bae et al., 2006), which is involved in the pathogenesis of RGD and aMCI (Butters et al., 2008). The finding further suggests that regional characteristics of the prefrontal regions may also be a valuable marker for distinguishing RGD from aMCI. Therefore, WM network analyses had sufficient sensitivity to identify the distinctions between aMCI and RGD patients. From connectivity-based analysis, we found two patient groups with distinct patterns of reduced connections (i.e., right-hemispheric asymmetries in the disrupted connected components in the RGD and bilateral deficits in the aMCI subjects). These findings are consistent with evidence that the right hemisphere is selectively involved in the processing of negative emotions (e.g., characterizing symptoms in depression) (Hecht, 2010) and that complicated circuits associated with the bilateral brain could provide significant discriminant features for the classification of patients with cognitive impairments (e.g., MCI) (Wee et al., 2012).

\section{Methodological issues}

There are several issues that should be addressed. First, the tracking procedure of deterministic tractography always terminates when it reaches regions with fiber crossings and low FA values

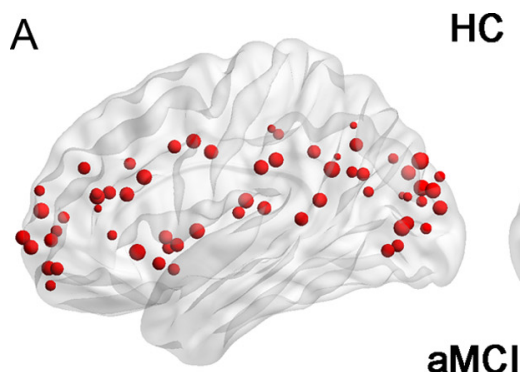

$\mathrm{HC}$
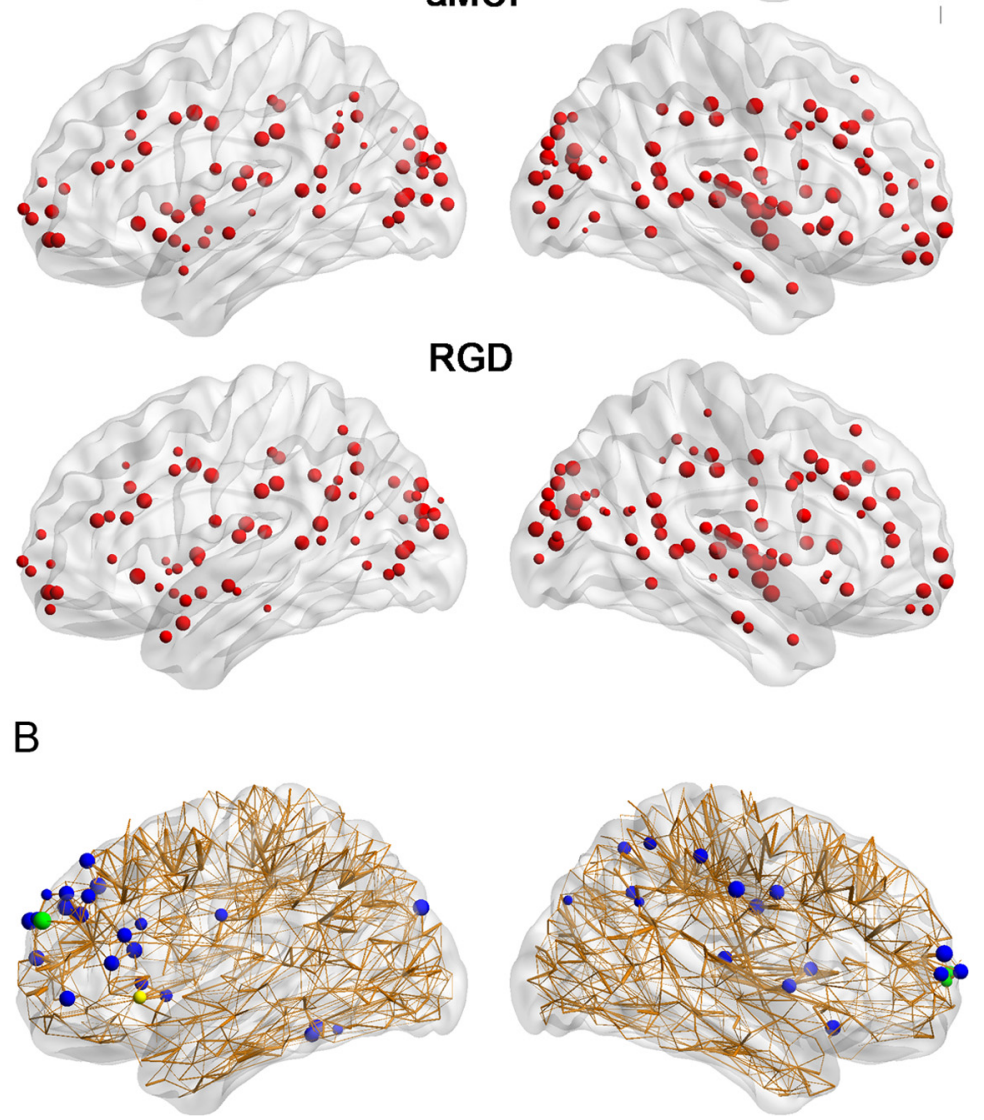

Figure 6. Hub distributions and regional differences of high-resolution networks (H-1024) in the $\mathrm{HC}$, aMCl, and RGD groups. $A$, Three-dimensional representations of the hub distributions in the control, aMCl, and RGD groups. The hub nodes are shown in red with the node sizes representing their nodal efficiency values. The regions were mapped onto the cortical surface at a lateral view. $\boldsymbol{B}$, The distribution of the nodes exhibited significant group effects in the nodal efficiency among the three groups at $p<$ 0.005 (uncorrected). The node sizes indicate the significance of between-group differences in the regional efficiency. Post hoc tests showed that most of the nodes ( 36 of 42) exhibited a reduced efficiency in both of the aMCI and RGD patients relative to the controls. Between the aMCI and RGD groups, three nodes in the prefrontal cortex (in green) have a reduced efficiency in the RGD patients than the aMCl patients. One node in the left inferior frontal gyrus (in yellow) showed a reduced efficiency in the aMCl patients compared with the RGD patients. The network shown here was constructed by averaging the anatomical connection matrices of all of the healthy controls with a threshold of a sparsity of $1 \%$. The nodes and connections were mapped onto the cortical surfaces using in-house BrainNet viewer software (www.nitrc.org/projects/bnv/).

because of the "fiber crossing" problem. This may result in a loss of existing fibers. Other studies have proposed the use of probabilistic tractography to define network edges (Iturria-Medina et al., 2008; Gong et al., 2009b), which could be helpful in addressing these issues. Second, we performed two parcellation methods (L-AAL and H-1024) to construct the WM network. The network analysis of the global properties showed comparable results. Nevertheless, there were also some discrepancies in the regional alterations between the aMCI and RGD groups. Such discrepancies may be driven by differences in the graph properties under the different node scales (Fornito et al., 2010; Zalesky et al., 2010a) or the subregions of some anatomical structures (e.g., PCG). There- 
fore, graph analyses with different spatial resolutions would be necessary and important to provide a more comprehensive picture of the topological alterations of brain networks in the patients. Third, the recruitment of the present aMCI subjects was based only on clinical criteria. Some of the subjects may not have displayed any underlying $\mathrm{AD}$ pathology and may represent a "contamination" of the sample with non-AD cases. This may be resolved by obtaining additional biomarker information to better characterize the study groups. This would further support the current diagnosis of "aMCI due to AD," as was recently published in the revised diagnostic criteria for AD (Dubois et al., 2010). Finally, the present three groups were not matched for age, albeit the fact that the age effect was removed in all of the network analyses. Therefore, these data should be interpreted with caution.

\section{References}

Achard S, Bullmore E (2007) Efficiency and cost of economical brain functional networks. PLoS Comput Biol 3:e17.

Alexopoulos GS, Murphy CF, Gunning-Dixon FM, Latoussakis V, Kanellopoulos D, Klimstra S, Lim KO, Hoptman MJ (2008) Microstructural white matter abnormalities and remission of geriatric depression. Am J Psychiatry 165:238-244.

Bae JN, MacFall JR, Krishnan KR, Payne ME, Steffens DC, Taylor WD (2006) Dorsolateral prefrontal cortex and anterior cingulate cortex white matter alterations in late-life depression. Biol Psychiatry 60:1356-1363.

Bai F, Zhang Z, Watson DR, Yu H, Shi Y, Yuan Y, Qian Y, Jia J (2009a) Abnormal integrity of association fiber tracts in amnestic mild cognitive impairment. J Neurol Sci 278:102-106.

Bai F, Zhang Z, Watson DR, Yu H, Shi Y, Yuan Y, Zang Y, Zhu C, Qian Y (2009b) Abnormal functional connectivity of hippocampus during episodic memory retrieval processing network in amnestic mild cognitive impairment. Biol Psychiatry 65:951-958.

Basser PJ, Pajevic S, Pierpaoli C, Duda J, Aldroubi A (2000) In vivo fiber tractography using DT-MRI data. Magn Reson Med 44:625-632.

Beason-Held LL (2011) Dementia and the default mode. Curr Alzheimer Res 8:361-365.

Blasko I, Kemmler G, Jungwirth S, Wichart I, Krampla W, Weissgram S, Jellinger K, Tragl KH, Fischer P (2010) Plasma amyloid beta-42 independently predicts both late-onset depression and Alzheimer disease. Am J Geriatr Psychiatry 18:973-982.

Bullmore E, Sporns O (2009) Complex brain networks: graph theoretical analysis of structural and functional systems. Nat Rev Neurosci 10:186-198.

Bullmore ET, Bassett DS (2011) Brain graphs: graphical models of the human brain connectome. Annu Rev Clin Psychol 7:113-140.

Butters MA, Whyte EM, Nebes RD, Begley AE, Dew MA, Mulsant BH, Zmuda MD, Bhalla R, Meltzer CC, Pollock BG, Reynolds CF 3rd, Becker JT (2004) The nature and determinants of neuropsychological functioning in late-life depression. Arch Gen Psychiatry 61:587-595.

Butters MA, Klunk WE, Mathis CA, Price JC, Ziolko SK, Hoge JA, Tsopelas ND, Lopresti BJ, Reynolds CF 3rd, DeKosky ST, Meltzer CC (2008) Imaging Alzheimer pathology in late-life depression with PET and Pittsburgh Compound-B. Alzheimer Dis Assoc Disord 22:261-268.

Conturo TE, Lori NF, Cull TS, Akbudak E, Snyder AZ, Shimony JS, McKinstry RC, Burton H, Raichle ME (1999) Tracking neuronal fiber pathways in the living human brain. Proc Natl Acad Sci USA 96:10422-10427.

Delbeuck X, Van der Linden M, Collette F (2003) Alzheimer's disease as a disconnection syndrome? Neuropsychol Rev 13:79-92.

Dubois B, Feldman HH, Jacova C, Cummings JL, Dekosky ST, BarbergerGateau P, Delacourte A, Frisoni G, Fox NC, Galasko D, Gauthier S, Hampel H, Jicha GA, Meguro K, O'Brien J, Pasquier F, Robert P, Rossor M, Salloway S, Sarazin M, et al. (2010) Revising the definition of Alzheimer's disease: a new lexicon. Lancet Neurol 9:1118-1127.

Fennema-Notestine C, McEvoy LK, Hagler DJ Jr, Jacobson MW, Dale AM; The Alzheimer's Disease Neuroimaging Initiative (2009) Structural neuroimaging in the detection and prognosis of pre-clinical and early $\mathrm{AD}$. Behav Neurol 21:3-12.

Fornito A, Zalesky A, Bullmore ET (2010) Network scaling effects in graph analytic studies of human resting-state FMRI data. Front Syst Neurosci 4:22.

Gong G, He Y, Concha L, Lebel C, Gross DW, Evans AC, Beaulieu C (2009a) Mapping anatomical connectivity patterns of human cerebral cortex using in vivo diffusion tensor imaging tractography. Cereb Cortex 19:524-536.

Gong G, Rosa-Neto P, Carbonell F, Chen ZJ, He Y, Evans AC (2009b) Ageand gender-related differences in the cortical anatomical network. J Neurosci 29:15684-15693.

Goveas J, Xie C, Wu Z, Douglas Ward B, Li W, Franczak MB, Jones JL, Antuono PG, Yang Z, Li SJ (2011) Neural correlates of the interactive relationship between memory deficits and depressive symptoms in nondemented elderly: resting fMRI study. Behav Brain Res 219:205-212.

Hagmann P, Cammoun L, Gigandet X, Meuli R, Honey CJ, Wedeen VJ, Sporns O (2008) Mapping the structural core of human cerebral cortex. PLoS Biol 6:e159.

He Y, Evans A (2010) Graph theoretical modeling of brain connectivity. Curr Opin Neurol 23:341-350.

He Y, Chen ZJ, Evans AC (2007) Small-world anatomical networks in the human brain revealed by cortical thickness from MRI. Cereb Cortex 17:2407-2419.

He Y, Chen Z, Evans A (2008) Structural insights into aberrant topological patterns of large-scale cortical networks in Alzheimer's disease. J Neurosci 28:4756-4766.

He Y, Chen Z, Gong G, Evans A (2009) Neuronal networks in Alzheimer's disease. Neuroscientist 15:333-350.

Hecht D (2010) Depression and the hyperactive right-hemisphere. Neurosci Res 68:77-87.

Humphries MD, Gurney K, Prescott TJ (2005) Is there an integrative center in the vertebrate brainstem? A robotic evaluation of a model of the reticular formation viewed as an action selection device. Adapt Behav 13:97-113.

Iturria-Medina Y, Sotero RC, Canales-Rodríguez EJ, Alemán-Gómez Y, Melie-García L (2008) Studying the human brain anatomical network via diffusion-weighted MRI and graph theory. Neuroimage 40:1064-1076.

Jack CR Jr, Lowe VJ, Senjem ML, Weigand SD, Kemp BJ, Shiung MM, Knopman DS, Boeve BF, Klunk WE, Mathis CA, Petersen RC (2008) ${ }^{11} \mathrm{C}$ PiB and structural MRI provide complementary information in imaging of Alzheimer's disease and amnestic mild cognitive impairment. Brain 131:665-680.

Jagust WJ, Bandy D, Chen K, Foster NL, Landau SM, Mathis CA, Reiman EM, Skovronsky D, Koeppe RA; Alzheimer's Disease Neuroimaging Initiative (2010) The Alzheimer's Disease Neuroimaging Initiative positron emission tomography core. Alzheimers Dement 6:221-229.

Kenny ER, Blamire AM, Firbank MJ, O'Brien JT (2012) Functional connectivity in cortical regions in dementia with Lewy bodies and Alzheimer's disease. Brain 135:569-581.

Latora V, Marchiori M (2001) Efficient behavior of small-world networks. Phys Rev Lett 87:198701.

Leistedt SJ, Coumans N, Dumont M, Lanquart JP, Stam CJ, Linkowski P (2009) Altered sleep brain functional connectivity in acutely depressed patients. Hum Brain Mapp 30:2207-2219.

Lo CY, Wang PN, Chou KH, Wang J, He Y, Lin CP (2010) Diffusion tensor tractography reveals abnormal topological organization in structural cortical networks in Alzheimer's disease. J Neurosci 30:16876-16885.

Maslov S, Sneppen K (2002) Specificity and stability in topology of protein networks. Science 296:910-913.

Milak MS, Parsey RV, Keilp J, Oquendo MA, Malone KM, Mann JJ (2005) Neuroanatomic correlates of psychopathologic components of major depressive disorder. Arch Gen Psychiatry 62:397-408.

Mori S, Crain BJ, Chacko VP, van Zijl PC (1999) Three-dimensional tracking of axonal projections in the brain by magnetic resonance imaging. Ann Neurol 45:265-269.

Morimoto SS, Gunning FM, Murphy CF, Kanellopoulos D, Kelly RE, Alexopoulos GS (2011) Executive function and short-term remission of geriatric depression: the role of semantic strategy. Am J Geriatr Psychiatry 19:115-122.

Petersen RC, Smith GE, Waring SC, Ivnik RJ, Tangalos EG, Kokmen E (1999) Mild cognitive impairment,clinical characterization and outcome. Arch Neurol 56:303-308. 
Pihlajamäki M, Jauhiainen AM, Soininen H (2009) Structural and functional MRI in mild cognitive impairment. Curr Alzheimer Res 6:179-185.

Rapp MA, Schnaider-Beeri M, Grossman HT, Sano M, Perl DP, Purohit DP, Gorman JM, Haroutunian V (2006) Increased hippocampal plaques and tangles in patients with Alzheimer disease with a lifetime history of major depression. Arch Gen Psychiatry 63:161-167.

Rubinov M, Sporns O (2010) Complex network measures of brain connectivity: uses and interpretations. Neuroimage 52:1059-1069.

Sexton CE, Kalu UG, Filippini N, Mackay CE, Ebmeier KP (2011) A metaanalysis of diffusion tensor imaging in mild cognitive impairment and Alzheimer's disease. Neurobiol Aging 32:2322.e5-e8.

Shu N, Liu Y, Li J, Li Y, Yu C, Jiang T (2009) Altered anatomical network in early blindness revealed by diffusion tensor tractography. PLoS One 4:e7228.

Shu N, Liu Y, Li K, Duan Y, Wang J, Yu C, Dong H, Ye J, He Y (2011) Diffusion tensor tractography reveals disrupted topological efficiency in white matter structural networks in multiple sclerosis. Cereb Cortex 21:2565-2577.

Sporns O (2011) The human connectome: a complex network. Ann N Y Acad Sci 1224:109-125.

Sporns O, Zwi JD (2004) The small world of the cerebral cortex. Neuroinformatics 2:145-162.

Stam CJ (2010) Characterization of anatomical and functional connectivity in the brain: a complex networks perspective. Int J Psychophysiol 77:186-194.

Stam CJ, Jones BF, Nolte G, Breakspear M, Scheltens P (2007) Small-world networks and functional connectivity in Alzheimer's disease. Cereb Cortex 17:92-99.

Supekar K, Menon V, Rubin D, Musen M, Greicius MD (2008) Network analysis of intrinsic functional brain connectivity in Alzheimer's disease. PLoS Comput Biol 4:e1000100.

Tzourio-Mazoyer N, Landeau B, Papathanassiou D, Crivello F, Etard O, Delcroix N, Mazoyer B, Joliot M (2002) Automated anatomical labeling of activations in SPM using a macroscopic anatomical parcellation of the MNI MRI single-subject brain. Neuroimage 15:273-289.

van den Heuvel MP, Stam CJ, Boersma M, Hulshoff Pol HE (2008) Smallworld and scale-free organization of voxel-based resting-state functional connectivity in the human brain. Neuroimage 43:528-539.

Wang J, Wang L, Zang Y, Yang H, Tang H, Gong Q, Chen Z, Zhu C, He Y (2009) Parcellation-dependent small-world brain functional networks: a resting-state fMRI study. Hum Brain Mapp 30:1511-1523.
Watts DJ, Strogatz SH (1998) Collective dynamics of "small-world " networks. Nature 393:440-442.

Wee CY, Yap PT, Zhang D, Denny K, Browndyke JN, Potter GG, WelshBohmer KA, Wang L, Shen D (2012) Identification of MCI individuals using structural and functional connectivity networks. Neuroimage 59:2045-2056.

Winblad B, Palmer K, Kivipelto M, Jelic V, Fratiglioni L, Wahlund LO, Nordberg A, Bäckman L, Albert M, Almkvist O, Arai H, Basun H, Blennow K, de Leon M, DeCarli C, Erkinjuntti T, Giacobini E, Graff C, Hardy J, Jack C, et al. (2004) Mild cognitive impairment-beyond controversies, towards a consensus: report of the international working group on mild cognitive impairment. J Intern Med 256:240-246.

Xie C, Goveas J, Wu Z, Li W, Chen G, Franczak MB, Jones JL, Zhang Z, Li SJ (2011) Neural basis of the association between depressive symptoms and memory deficits in nondemented subjects: resting-state fMRI study. Hum Brain Mapp. Advance online publication. Retrieved February 13, 2012. doi: $10.1002 / \mathrm{hbm} .21291$.

Yuan Y, Zhu W, Zhang Z, Bai F, Yu H, Shi Y, Qian Y, Liu W, Jiang T, You J, Liu Z (2008a) Regional gray matter changes are associated with cognitive deficits in remitted geriatric depression: an optimized voxel-based morphometry study. Biol Psychiatry 64:541-544.

Yuan Y, Zhang Z, Bai F, Yu H, Shi Y, Qian Y, Liu W, You J, Zhang X, Liu Z (2008b) Abnormal neural activity in the patients with remitted geriatric depression: a resting-state functional magnetic resonance imaging study. J Affect Disord 111:145-152.

Yuan Y, Hou Z, Zhang Z, Bai F, Yu H, You J, Shi Y, Liu W, Jiang T (2010) Abnormal integrity of long association fiber tracts is associated with cognitive deficits in patients with remitted geriatric depression: a crosssectional, case-control study. J Clin Psychiatry 71:1386-1390.

Zalesky A, Fornito A, Harding IH, Cocchi L, Yücel M, Pantelis C, Bullmore ET (2010a) Whole-brain anatomical networks: does the choice of nodes matter? Neuroimage 50:970-983.

Zalesky A, Fornito A, Bullmore ET (2010b) Network-based statistic: identifying differences in brain networks. Neuroimage 53:1197-1207.

Zhang J, Wang J, Wu Q, Kuang W, Huang X, He Y, Gong Q (2011) Disrupted brain connectivity networks in drug-naive, first-episode major depressive disorder. Biol Psychiatry 70:334-342.

Zhuang L, Wen W, Zhu W, Trollor J, Kochan N, Crawford J, Reppermund S, Brodaty H, Sachdev P (2010) White matter integrity in mild cognitive impairment: A tract-based spatial statistics study. Neuroimage 53:16-25. 